\title{
Evolving Probabilistically Significant Epistatic Classification Rules for Heterogeneous Big Datasets
}

\author{
John P Hanley \\ University of Vermont \\ 43 Colchester Ave \\ 206 Perkins Hall \\ 1-802-922-5175 \\ jhanley@uvm.edu
}

\author{
Jeffrey S Buzas \\ University of Vermont \\ 16 Colchester Ave \\ 201 Henry Lord House \\ 1-802-656-2971 \\ jeff.buzas@uvm.edu
}

\author{
Margaret J Eppstein \\ University of Vermont \\ 33 Colchester Ave \\ 327 Votey Hall \\ 1-802-656-1918 \\ maggie.eppstein@uvm.edu
}

\author{
Donna M Rizzo \\ University of Vermont \\ 33 Colchester Ave \\ 213 Votey Hall \\ 1-802-656-1495 \\ drizzo@uvm.edu
}

\begin{abstract}
We develop an algorithm to evolve sets of probabilistically significant multivariate feature interactions, with co-evolved feature ranges, for classification in large, complex datasets. The datasets may include nominal, ordinal, and/or continuous features, missing data, imbalanced classes, and other complexities. Our agelayered evolutionary algorithm generates conjunctive clauses to model multivariate interactions in datasets that are too large to be analyzed using traditional methods such as logistic regression. Using a novel hypergeometric probability mass function for fitness evaluation, the algorithm automatically archives conjunctive clauses that are probabilistically significant at a given threshold, thus identifying strong complex multivariate interactions. The method is validated on two synthetic epistatic datasets and applied to a complex real-world survey dataset aimed at determining the drivers of household infestation for an insect that transmits Chagas disease. We identify a set of 178,719 predictive feature interactions that are associated with household infestation, thus dramatically reducing the size of the search space for future analysis.
\end{abstract}

\section{Keywords}

Hypergeometric PMF; epistasis; multivariate analysis; Chagas

\section{INTRODUCTION}

This work was motivated by the desire to mine data from a large socioeconomic survey aimed at identifying the drivers of household infestation with an insect that transmits Chagas disease, which if untreated is life-threatening. The disease is transmitted by insects in the subfamily Triatomine [3]. Approximately 110 million people live where the disease is endemic and of those, approximately 8 million are infected [10]. To decrease the risk of disease

Permission to make digital or hard copies of all or part of this work for personal or classroom use is granted without fee provided that copies are not made or distributed for profit or commercial advantage and that copies bear this notice and the full citation on the first page. Copyrights for components of this work owned by others than ACM must be honored. Abstracting with credit is permitted. To copy otherwise, or republish, to post on servers or to redistribute to lists, requires prior specific permission and/or a fee. Request permissions from Permissions@acm.org.

GECCO '16, July 20-24, 2016, Denver, CO, USA

(C) 2016 ACM. ISBN 978-1-4503-4206-3/16/07 ...\$15.00

DOI: http://dx.doi.org/10.1145/2908812.2908931 transmission, mitigation strategies, known as Ecohealth interventions, have been implemented to lessen the chance of household infestation of the Triatomine vectors by removing known hiding locations [15]. However, many areas where the disease is endemic have limited resources for these preventative household improvements. Detailed entomologic and socioeconomic surveys are conducted to try to identify the drivers of infestation [1] in order to determine the most cost-effective strategies to reduce infestation. Mining these complex Chagas survey datasets for useful information has proven to be a major challenge, due to a variety of factors, including missing data, imbalanced class outcomes, heterogeneity of drivers of infestation, non-independence of some features, and complex, possibly highorder, nonlinear interactions between many of the potential predictive features. Traditional statistical methods for survey analysis, such as logistic regression, are not suitable for analysis of the Chagas survey data because $p$-values are unreliable in large datasets [13] such as this.

The goal of this work is the development of a method to identify probabilistically significant epistatic interactions among input features correlated with a desired categorical outcome, where the input features may be comprised of multiple data types (e.g., nominal, ordinal, and continuous). For example, when the insect infests a house, it needs a food source and habitat (place to hide) at minimum. In addition, researchers believe that other factors (e.g., lights, windows) impact the attraction of the insect to the house. So in addition to habitat and food sources, the survey adds risk factors that suggest more interesting epistatic interactions of a higher order.

As a first step in the analysis of the Chagas survey dataset, our methodology is intended to deal with the massive number of complex multivariate interactions present in big datasets. A number of studies assume that individual features have main effects and use univariate logistic regression as a feature selection tool to reduce the size of the feature set that is to be mined for higher order interactions $[4,9]$. Decision trees similarly enforce a hierarchical structure [17] based on the presence of main effects. Using main effects as a filter, however, precludes discovery of purely epistatic interactions that may drive a system, which may be the case for complex diseases [21], and our Chagas survey dataset. 
We first tried applying ExSTraCS v1.0, a Michigan-style Learning Classifier System (LCS) specifically designed to search for heterogeneous epistatic interactions [19], to our dataset. To our knowledge, ExSTraCS is the best available method for analyzing large datasets when output categories are associated with more than one heterogeneous multivariate feature combination. However, we discovered that ExSTraCS was not well-suited to our Chagas survey dataset. Samples with too much missing data were problematic and needed to be removed, therefore causing us to throw away portions of our survey data. Also, our unbalanced dataset seemed to negatively impact results. In addition, ExSTraCS, as in most Michigan-style learning classifier systems [2], learns incrementally from individual samples rather than applying batch learning, thus requiring computationally expensive post-processing permutation tests to evaluate the significance of the identified features [20]. Furthermore, in our Chagas survey data, there may be ranges or sets of values for each input feature (i.e., predictor variable) that are associated with the same output category. Co-evolving feature ranges is not explicitly supported by ExSTraCS, but has been used in a few other genetics-based, machine-learning algorithms. For example, [14] and [17] supported co-evolution of ranges of feature values for continuous and ordinal features in conjunctive clauses (i.e., features with a range joined by ANDs), and [5, 6] supported coevolution of feature set values for nominal features in conjunctive clauses.

Determining the statistical significance of evolved interaction terms is challenging in large datasets, and has been approached in various ways. As mentioned above, one option is to run postprocessing permutation tests [20]. Others have tried to build significance tests directly into the fitness function. In [14], the authors propose two fitness functions for evaluating the conjunctive clauses. The first is the product of the accuracy and the coverage of the conjunctive clause; but this does not provide a natural threshold to identify important conjunctive clauses. The second, referred to as a CBayesian function, is the product of probabilities that, given the target output state, each evolved predictor variable has the evolved range. While this allows one to set a threshold for significance, it is based on a product of probabilities of univariate associations with the target class rather than on the probability of the multivariate association. Others $[17,18]$ use a statistics based fitness function using the z-score of the standard normal distribution that takes the following inputs: (1) coverage of the conjunctive clause, (2) proportion of observations with the target outcome, and (3) the number of observations matching both the conjunctive clause and the target outcome divided by the number of observations that match the conjunctive clause. The benefit of using a standard normal distribution is that $p$-value thresholds (e.g., $p<0.05$ ) may be applied to the fitness values to identify statistically significant conjunctive clauses. However, $p$-values decrease inversely with the size of the dataset, making the $p$-value an unreliable statistic for "Big Data" applications [13].

To overcome this challenge, we propose using the hypergeometric probability mass function (PMF) [12] as a fitness function. The hypergeometric PMF may be thought of as a pseudo-Bayesian equation. One benefit of using the probability derived from the hypergeometric PMF is that it takes into account the size of the dataset and the distribution of the output categories. Therefore, unlike in the method of $[17,18]$, the ratio of output categories does not need to be balanced. Also, since the fitness is a probability, the user can choose a threshold that is easily interpreted and can compare probabilities below the threshold.
In Section 2, we describe an evolutionary algorithm (EA) to efficiently identify sets of probabilistically significant conjunctive clauses that represent multivariate interactions in large datasets. We first define the hypergeometric PMF fitness function, and then discuss how we incorporated this fitness function into an evolutionary algorithm using age-layers and an archive. In Section 3 we describe two synthetic epistatic datasets used for validation as well as the complex real-world survey dataset aimed at finding drivers of household infestation for an insect that transmits Chagas disease. We show results of these experiments in Section 4, and present concluding remarks in Section 5.

The primary intended contribution of this paper is a method to find collections of conjunctions with significant associations to a target class, thereby dramatically reducing the size of the search space for subsequent analysis in Big Data classification problems. How one chooses to subsequently analyze that set will depend on the nature and complexity of the dataset and the stakeholder's objectives. For the relatively simple synthetic validation datasets, we illustrate how our genetic algorithm identifies a minimal disjunctive combination of these archived conjunctive clauses (i.e., conjunctive clauses joined by ORs), which simultaneously maximize coverage and accuracy. However, secondary mining of the large set of significant conjunctive clauses identified in the complex real world Chagas dataset will require more analyses, a topic we leave for future research.

\section{ALGORITHMIC METHODS 2.1 Fitness Function}

We seek sets of conjunctive clauses that have a probabilistically significant association with a certain class outcome $k$ (e.g., infestation or disease). We define the fitness of a given conjunctive clause as follows:

Fitness (conjunctive clause $)=\frac{\left(\begin{array}{c}X_{t o t} \\ x_{\text {match }}\end{array}\right)\left(\begin{array}{c}N_{\text {tot }}-X_{\text {tot }} \\ n_{\text {match }}-x_{\text {match }}\end{array}\right)}{\left(\begin{array}{c}N_{\text {tot }} \\ n_{\text {match }}\end{array}\right)}$

where, $N_{t o t}=$ is the total number of samples in the dataset,

$X_{t o t}$ is the total number of those samples in the target class $k$,

$n_{\text {match }}$ is the number of samples whose features match a given clause, and $x_{\text {match }}$ is the number of observations that match the clause and are in target class $k$. Eq. (1) is a hypergeometric PMF [12] and quantifies the likelihood that the observed association between the clause and the target class is due to chance; thus, lower values of this fitness function are indicative of potential association between a clause and a target class.

We develop an evolutionary algorithm (described in the Section 2.4) to find clauses with low fitness (per Eq. 1), and archive all clauses whose fitness is less than a specified probability threshold $T$. In the studies reported here, we used $T=10^{-9}$, which translates to a probability of 1 in a billion that a clause with fitness $=T$ is randomly associated with the target class $k$.

\subsection{Evolving Conjunctive Clauses}

To support the co-evolution of feature sets and feature ranges, we represent multivariate interactions with conjunctive clauses of the following form:

$$
P V_{i} \in a_{i} \wedge P V_{j} \in a_{j} \ldots \wedge P V_{k} \in a_{k}
$$

where each $P V_{i}$ represents a predictor variable $i$ that may be nominal, ordinal, or continuous, and whose value lies in $a_{i}$, a 
specified range or set of values. The population is stored using a combination of a binary matrix (indicating feature presence/absence) and a parallel cell array (specifying the range or set of values for each feature present) (Figure 1). In this work, we allow the number of features present in a given clause to range from 1 to $L$ (the number of features); we take this conservative approach in order to avoid a priori assumptions on the maximum order of interactions present in a given complex system.

The evolutionary algorithm is summarized in the pseudo code shown in Algorithm 1, and is described below. Line 1 of Algorithm 1 states that the evolutionary algorithm is run separately for each desired target outcome class $k$, out of up to $K$ possible classes. Lines 2-9 describe our implementation of the Age-Layer Population Structure (ALPS) created by [11]. We add a novel population $\left(\operatorname{Pop}_{N}\right)$ every 10 generations $\left(G n_{N}\right)$. In total, our algorithm has 10 "Age-Layers," however, layers $1-9$ follow the structure described in [11]; where the maximum age for layers 1,2, $\ldots, 8$ is $10,20, \ldots, 80$, respectively, and the ninth layer has no age limit. Our tenth layer is used to archive the population of conjunctive clauses $\left(P o p_{A}\right)$ that have a fitness $<T$ described in lines $10-16$ of Algorithm 1, and has no size limit. Only conjunctive clauses that have a proportion $\left(x_{\text {match }} / n_{\text {match }}\right)$ of the current output class that is greater or equal to the global proportion $\left(X_{t o t} / N_{t o t}\right)$ are retained. This biases the algorithm toward conjunctive clauses that are useful in finding associations to a given class $k$.

Each generation, duplicate conjunctive clauses are removed from both the archived and non-archived populations (Algorithm 1, line
17). The only other time conjunctive clauses are removed from the population is if the maximum size of the non-archived population $\left(P_{o p_{N A}}\right)$ is exceeded. If this occurs, then for each non-archived age layer, only the two most-fit conjunctive clauses for each feature variable are saved for the next generation. These two most-fit rules are selected one feature at a time to maintain diversity in the population. When selecting most-fit rules without accounting for feature representation, we observed that some more complex features (e.g., larger ranges) and interactions did not evolve to the desired signal. Repeat conjunctive clauses are prevented by selection without replacement. To avoid bias, the order of features considered is randomized in this process.

Algorithm 2, describes the creation of novel populations in the first layer $\left(\operatorname{Pop}_{N}\right)$. Line 2 states that an observation $n^{z}$ is randomly selected from the observations equal to the target outcome $k$. All random numbers were generated from a uniform distribution. Each conjunctive clause is created as follows. We randomly select the number of features to be present in the clause (Algorithm 2, Line 3 ), and then randomly select the features for the clause. If the selected feature is continuous or ordinal, the upper and lower bounds of the conjunctive clause for the feature variable are set to the corresponding value in the randomly selected observation $n^{z}$ (Algorithm 2, Lines 6-7). For nominal features, the value is set to the corresponding value of $n^{z}$. At no point can the conjunctive clause contain blank ranges or sets for selected features.

Algorithm 3 describes mutation and crossover for the evolutionary algorithm. In each generation, all the conjunctive clauses in $P_{o p_{N A}}$ undergo either mutation or crossover. However, for the archive $\left(\mathrm{Pop}_{A}\right)$ only up to two randomly selected conjunctive clauses for

\begin{tabular}{|c|c|c|c|c|c|c|c|c|}
\hline \multicolumn{9}{|c|}{$(\mathbf{A})$ Input Features Present } \\
\hline Nominal & Continuous & Nominal & Ordinal & Ordinal & Nominal & Nominal & $\ldots$ & Ordinal \\
\hline $\mathbf{P V}_{\mathbf{1}}$ & $\mathbf{P V}_{\mathbf{2}}$ & $\mathbf{P V}_{\mathbf{3}}$ & $\mathbf{P V}_{\mathbf{4}}$ & $\mathbf{P V}_{\mathbf{5}}$ & $\mathbf{P V}_{\mathbf{6}}$ & $\mathbf{P V}_{\mathbf{7}}$ & $\ldots$ & $\mathbf{P V}_{\mathbf{L}}$ \\
\hline 1 & 0 & 0 & 0 & 1 & 1 & 0 & $\ldots$ & 0 \\
\hline 0 & 1 & 0 & 0 & 0 & 0 & 0 & $\ldots$ & 0 \\
\hline 0 & 0 & 1 & 0 & 1 & 0 & 1 & $\ldots$ & 1 \\
\hline 1 & 1 & 1 & 1 & 1 & 1 & 1 & $\ldots$ & 1 \\
\hline 1 & 1 & 0 & 1 & 0 & 1 & 0 & $\ldots$ & 0 \\
\hline 1 & 0 & 1 & 0 & 1 & 0 & 1 & $\ldots$ & 1 \\
\hline
\end{tabular}

\begin{tabular}{|c|c|c|c|c|c|c|c|c|}
\hline \multicolumn{7}{|c|}{ (B) Ranges or Sets of Values of Corresponding Features } \\
\hline Nominal & Continuous & Nominal & Ordinal & Ordinal & Nominal & Nominal & $\ldots$ & Ordinal \\
\hline $\mathbf{P V}_{\mathbf{1}}$ & $\mathbf{P V}_{\mathbf{2}}$ & $\mathbf{P V}_{\mathbf{3}}$ & $\mathbf{P V}_{\mathbf{4}}$ & $\mathbf{P V}_{\mathbf{5}}$ & $\mathbf{P V}_{\mathbf{6}}$ & $\mathbf{P V}_{\mathbf{7}}$ & $\boldsymbol{\cdots}$ & $\mathbf{P V}_{\mathbf{L}}$ \\
\hline$[2,4]$ & {[]} & {[]} & {[]} & {$[2-4]$} & {$[3]$} & {[]} & $\cdots$ & {[]} \\
\hline[] & {$[0.5-1.5]$} & {[]} & {[]} & {[]} & {[]} & {[]} & $\cdots$ & {[]} \\
\hline[] & {[]} & {$[2]$} & {[]} & {$[3-3]$} & {[]} & {$[1,2,4,5]$} & $\ldots$ & {$[2-6]$} \\
\hline$[1,3]$ & {$[7.5-10.0]$} & {$[2]$} & {$[1-2]$} & {$[1-3]$} & {$[1,4,5]$} & {$[4]$} & $\ldots$ & {$[1-4]$} \\
\hline$[2,4]$ & {$[3.5-10.0]$} & {[]} & {$[3-3]$} & {[]} & {$[3,4]$} & {[]} & $\ldots$ & {[]} \\
\hline$[1,2,3]$ & {[]} & {$[1]$} & {[]} & {$[1-3]$} & {[]} & {$[1,4]$} & $\ldots$ & {$[2-2]$} \\
\hline
\end{tabular}

Figure 1. The conjunctive clauses are stored using a combination of a binary matrix (A) and a parallel cell array (B), where each row corresponds to one conjunctive clause of predictor variables (PV); a one in the binary matrix indicates the feature is present in the conjunctive clause; and the entry in the corresponding location of the cell array represents the feature range or set of values. 


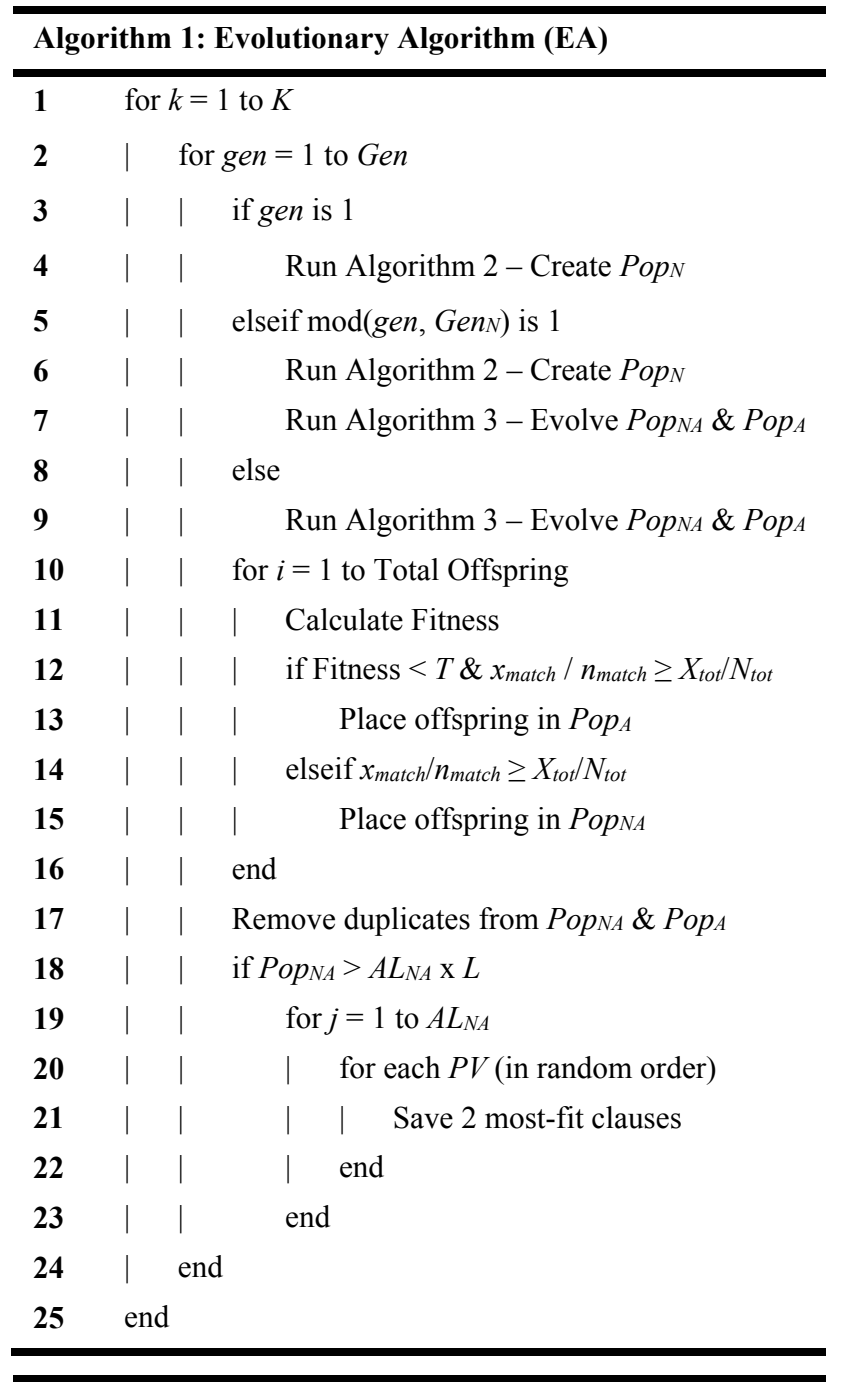

\begin{tabular}{|c|c|c|}
\hline \multicolumn{3}{|c|}{ Algorithm 2: Initialize Novel Populations } \\
\hline 1 & for $i=$ & to $2 \times L$ \\
\hline 2 & I $\mathrm{R}$ & domly select $n^{z}$ where $n^{z}=k$ \\
\hline 3 & $\mathrm{Se}$ & ect order of conjunctive clause ( 1 to $L$ ) \\
\hline 4 & fo & $j=1$ to order \\
\hline 5 & | 1 & Randomly select (without replacement) $P V_{y}$ \\
\hline 6 & $1 \mid$ & if $P V_{y}$ is ordinal or continuous \\
\hline 7 & $1 \mid$ & Set bounds to $P V_{y}^{Z}$ \\
\hline 8 & I en & \\
\hline 9 & end & \\
\hline
\end{tabular}

each $P V$ undergo mutation or crossover each generation. This is a result of there being no limit on the population size of Pop $_{A}$. When testing the algorithm on the [21] dataset, we found that the probability of crossover $P r_{x}=0.25$ consistently enabled us to find all of the "true" bivariate interactions, so we used this rate for the other datasets as well. If a conjunctive clause is selected to undergo crossover, a second parent is randomly selected, using tournament selection with replacement and a tournament size of 3 , from either the current age-layer or the adjacent younger age-layer, if one

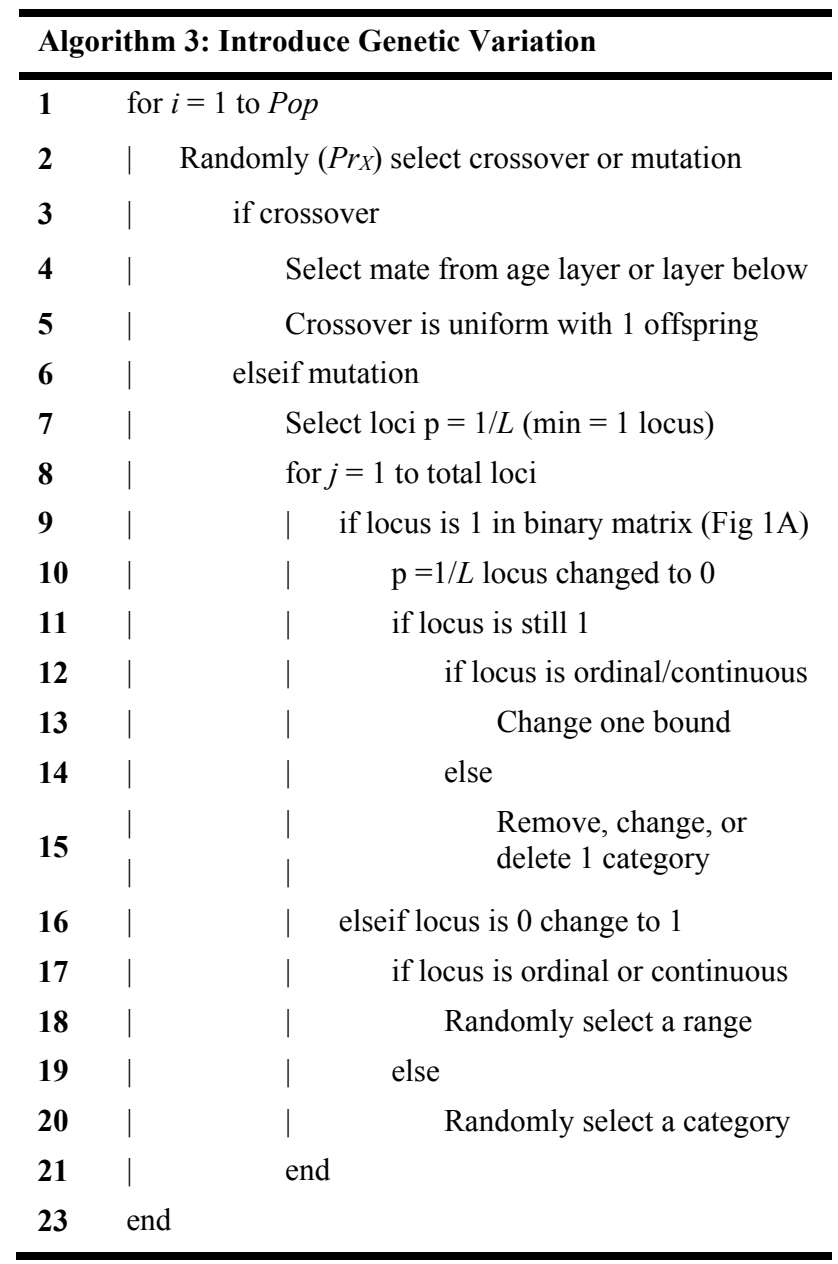

exists. The parents then undergo uniform crossover and produce one offspring (Algorithm 3, Lines 3-5).

If a conjunctive clause is selected to undergo mutation, then the probability that a given feature will undergo mutation is $p_{m}=1 / L$, a standard rule of thumb [8], although we force at least one feature to be selected (Algorithm 3, Line 7). If the selected feature value is 1 in the binary matrix (Figure $1 \mathrm{~A}$ ), then there is a $1 / L$ probability that the feature will be changed to zero in the corresponding binary matrix, reducing the order of the conjunctive clause (Algorithm 3, Lines $9-10$ ). If the locus is not changed to zero in the binary matrix, then the set of the locus is changed. For continuous or ordinal features, either the lower or upper bound is randomly changed to a value within the range of the feature, but ensuring that the lower bound is $\leq$ the upper bound (Algorithm 3, Lines 12-13). We also ensure that the upper and lower bounds never span the entire range of possible values for a given feature, as this would carry no information. If the locus is nominal, then one category from the set is randomly added, changed, or deleted from the set of possible feature values (Algorithm 3, Lines $14-15$ ). We also ensure the set of possible values is never empty nor ever contains all possible values for that feature.

If the selected feature value is zero in the binary matrix (Figure 1A), then the value is set to 1 . For nominal, continuous, or ordinal features, a range is randomly selected within the range of the current feature. For nominal features a category is selected at random (Algorithm 3, Lines 16-20). While this may appear to bias the algorithm to higher order interactions, the probability that a 
newly added feature will create a fit offspring (and thus be retained) is small.

The values for control parameters of this study (Table 1) were selected as a combination of published recommendations in the literature and parameters that were empirically determined as the most effective at quickly identifying the 4 true conjunctions in the first validation dataset (such as the probability of crossover $\operatorname{Pr}_{x}$ ), described earlier.

Table 1. EA parameters used of evolving conjunctions.

\begin{tabular}{|l|c|}
\hline Parameter & Value \\
\hline Total \# of Predictor Variables $(L)$ & Dataset dependent \\
\hline Threshold $(T)$ & $10^{-9}$ \\
\hline \# Non-Archived Age Layers $\left(A L_{N A}\right)$ & 9 \\
\hline Novel Population $\left(\right.$ Pop $\left._{N}\right)$ & $2 \times L$ \\
\hline Non-Archive Pop. $\left(\right.$ Pop $\left._{N A}\right)$ & Max $=A L_{N A} \times L$ \\
\hline Archive Population $\left(P_{o p_{A}}\right)$ & Max $=\infty$ \\
\hline Generations $(G e n)$ & Dataset dependent \\
\hline Generations Until Novel Pop $\left(G e n_{N}\right)$ & 10 \\
\hline Crossover Function $\left(F_{X}\right)$ & Uniform \\
\hline Probability of Crossover $\left(\operatorname{Pr}_{X}\right)$ & 0.25 \\
\hline Mutation Function $\left(F_{M}\right)$ & $\left\{\right.$ uniform, $\left.p_{m}=1 / L\right\}$ \\
\hline Crossover Mate Selection & $\{$ tournament, size $=3\}$ \\
\hline \# Archive Offspring $(O f f A)$ & $2 \times L$ \\
\hline
\end{tabular}

\subsection{Evolving DNF Rules}

After sets of probabilistically significant conjunction clauses have been identified using Algorithm 1, subsequent mining of this reduced search space can reveal useful combinations of these conjunctions. For example, by treating each conjunction in the archive as a binary feature, one can use a genetic algorithm (GA) to identify disjunctive combinations of these features (i.e., clauses in disjunctive normal form, or DNF). DNFs are thus represented as bit strings, where bit values represent the presence or absence of each conjunctive clause from the archived dataset. For this step, we defined fitness of a clause as follows:

DNF_Fitness $($ clause $)=\overline{A c c} \times \overline{\operatorname{Cov}} \times$ NonRep $\times$ TotCov

Where $\overline{A c c}$ is the weighted average accuracy $\left(\Sigma x_{\text {match }} / \Sigma n_{\text {match }}\right)$ of the conjunctive clauses, $\overline{\operatorname{Cov}}$ is the average coverage ( $\left.x_{\text {match }} / X_{\text {tot }}\right)$ of the conjunctive clauses, NonRep is the number of samples with output $=k$ that conjunctive clauses in the DNF collectively cover divided by the sum of the number of samples with output $=k$ each conjunctive clause individually covers, and TotCov is the number of samples with output $=k$ that conjunctive causes collectively cover divided by the total number of observations in the dataset with output $=k$. The GA was trying to maximize the fitness defined in Eq. (3).

The GA is based on the Age-Fitness Pareto Optimization developed by [16], with pseudo code shown in Algorithm 4 and control parameters in Table 2. In the first generation, a population of 20 DNFs is generated by randomly selecting between $1-20$ conjunctive clauses to be present (Algorithm 4, Line 3). Each DNF is evaluated using the fitness function Eq. (3); and each DNF is

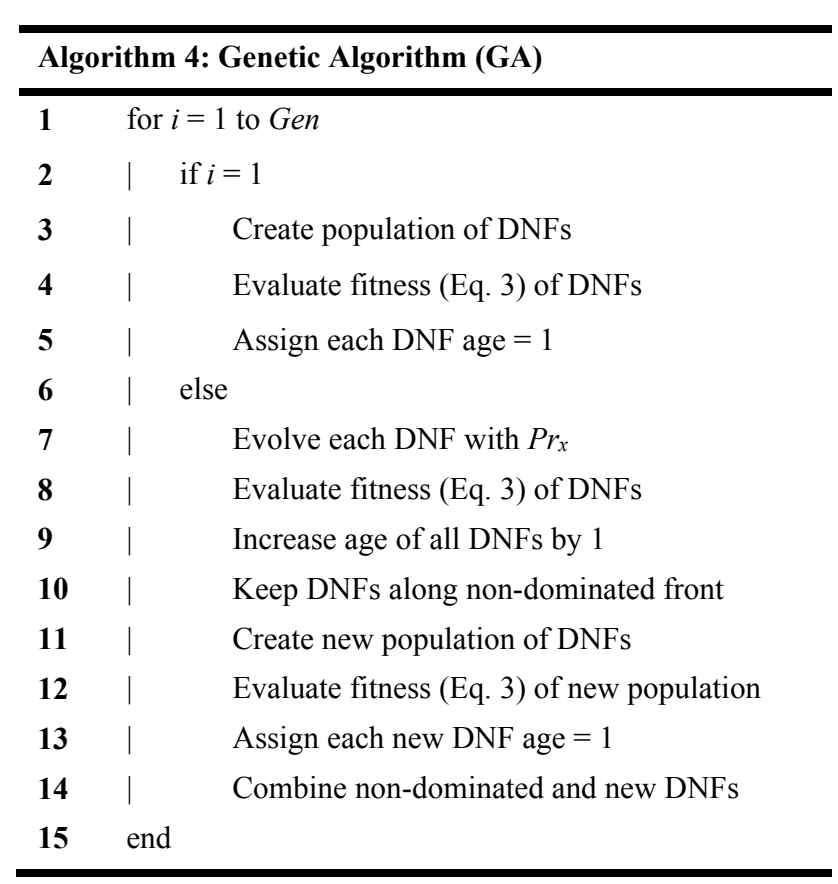

Table 2. GA parameters used for Evolving DNFs.

\begin{tabular}{|l|c|}
\hline Parameter & Value \\
\hline Total \# of Conjunctive Clauses $(L)$ & Dataset dependent \\
\hline Generations $(G e n)$ & Dataset dependent \\
\hline Probability of Crossover $\left(P r_{x}\right)$ & 0.75 \\
\hline Mutation Function $\left(F_{M}\right)$ & $\left\{\right.$ uniform, $\left.p_{m}=1 / L\right\}$ \\
\hline Crossover Mate Selection & $\{$ tournament, size $=3\}$ \\
\hline Crossover Function $\left(F_{X}\right)$ & Union and Intersection \\
\hline Probability of Union $\left(P_{u}\right)$ & 0.5 \\
\hline New Population Size $\left(\operatorname{Size}_{\text {Pop }}\right)$ & 20 \\
\hline Max \# Initial of Clauses $\left(\right.$ Max $\left._{c c}\right)$ & 20 \\
\hline
\end{tabular}

assigned an age $=1$ (Algorithm 4 Lines $4-5$ ). For subsequent generations, each DNF undergoes either mutation or crossover with $\operatorname{Pr}_{x}$ (Algorithm 4, Line 7). DNFs selected for mutation will have a $p_{m}$ of having each bit flipped consequently adding and removing conjunctive clauses. All offspring derived from mutation are given the same age as the parent. DNFs selected for crossover undergo either set union or set intersection $\left(\mathrm{Pr}_{u}\right)$ [7]. The second parent is randomly selected, using tournament selection with replacement and a tournament size of 3 . If two parents do not have a single conjunctive clause in common then crossover is forced to be union. All offspring are given the age of the oldest parent. Offspring DNFs are then evaluated using the fitness function (Eq. 3) (Algorithm 4, Line 8) and the age of both offspring and parents will increase by one (Algorithm 4, Line 9). The offspring and parents are plotted based on their age and fitness and only those DNFs that lie along the non-dominated front, where dominance is determined by highest fitness and lowest age, are archived for the next generation (Algorithm 4, Line 10). A new population of 20 DNFs is generated by randomly selecting $1-20$ conjunctive clauses (Algorithm 4, Line 11). The fitness from Eq. (3) of the new DNFs is evaluated 
and all new DNFs are assigned an age of 1 (Algorithm 4, Lines 12 $-13)$. The non-dominated DNFs are combined with the new DNFs and evolved in the subsequent generation.

\section{Test Datasets and Experiments}

We applied the evolutionary algorithm to two synthetic datasets for validation, and for preliminary analysis of the Chagas dataset.

\subsubsection{Synthetic Dataset 1}

The first validation dataset was designed by [21] to represent a synthetic Single Nucleotide Polymorphism (SNP) gene association dataset for a complex disease that incorporates both genetic heterogeneity and epistasis. This balanced dataset contains 1,600 samples each with 20 input feature variables, with exactly half of the samples associated with the "disease". Each input feature represents a SNP and is a ternary representation of whether the SNP is homozygous major, heterozygous, or homozygous minor. The synthetic dataset was designed such that no features had significant main effects and that four bivariate feature interactions (between two sets of two features) had strong correlations to different subsets of "diseased" observations, as shown in Table 3.

Table 3. The 4 "true" 2-way interactions in the [21] dataset (synthetic dataset 1). The last three columns contain the accuracy, coverage, and fitness of the rules.

\begin{tabular}{|c|c|c|c|c|c|c|c|}
\cline { 2 - 8 } \multicolumn{1}{l|}{} & $\mathbf{P V}_{\mathbf{0}}$ & $\mathbf{P V}_{\mathbf{1}}$ & $\mathbf{P V}_{\mathbf{2}}$ & $\mathbf{P V}_{\mathbf{3}}$ & Acc. & Cov. & Fit. \\
\hline $\mathbf{1}$ & 0 & 1 & - & - & $72 \%$ & $27 \%$ & $1.1 \times 10^{-17}$ \\
\hline $\mathbf{2}$ & 1 & 0 & - & - & $74 \%$ & $23 \%$ & $5.7 \times 10^{-17}$ \\
\hline $\mathbf{3}$ & - & - & 0 & 1 & $66 \%$ & $28 \%$ & $4.2 \times 10^{-12}$ \\
\hline $\mathbf{4}$ & - & - & 1 & 0 & $71 \%$ & $21 \%$ & $8.7 \times 10^{-13}$ \\
\hline
\end{tabular}

For this dataset, we ran 50 repetitions of the evolutionary algorithm (Algorithm 1) for 1,000 generations to try to find associations with the "diseased" class. We recorded the number of generations and fitness evaluations for each generation as well as the generation in which all the known a priori interactions were found. Afterwards, the archived conjunctive clauses were run through the GA (Algorithm 4) to find the "best" disjunctive normal form (DNF) of the archived rules. The GA was run 50 times; for each run we recorded the total number of generations and fitness evaluations to find the four "best" DNF rules in (Table 3) in the dataset associated with "diseased" observations.

\subsubsection{Synthetic Dataset 2}

While synthetic dataset 1 tested the algorithm's ability to identify the four correct bivariate epistatic interactions in this balanced dataset, it did not enable testing of the performance in the face of other complexities present in our Chagas dataset. We thus designed a second synthetic dataset to have 1) nominal and discrete input features, 2) higher-order epistatic interactions, 3) missing data, 4) heterogeneous associations with the same output class, and 5) imbalanced class outcomes. Specifically, the dataset consists of 25 input features, of which 17 are nominal (with values ranging from 1 to $3,4,6$, or 9) and 8 are discrete integers (with values ranging from 1 to 15 or 20). There are 1,000 simulated samples, of which 339 have output class $=1$, representing "diseased," and the remaining 661 have output class $=0$, representing "not diseased." Of the "diseased" samples, 144 were designed with a 4-way interaction (between 2 nominal and 2 discrete features) that is strongly correlated with disease, and a separate set of 195 samples have a 3-way interaction (between 3 nominal features) that is strongly correlated with the disease, as shown in Table 4. After randomly removing $20 \%$ of the data (to simulate missing data), there remained 58 4-way and 89 3-way intact interactions associated with class 1. Both "true" conjunctions had $100 \%$ accuracy but different levels of coverage and fitness (Table 4). The remaining 16 features contained randomly selected values.

Table 4. The 2 "true" 3-way and 4-way interactions built into synthetic dataset 2 . The Ns and Ds below each feature stand for nominal and discrete feature types, respectively. Also included is the range of each of the features in the true interactions. The last three columns contain the accuracy, coverage, and fitness of the rules.

\begin{tabular}{|c|c|c|c|c|c|c|c|c|c|c|}
\hline & $\mathbf{P V}_{0}$ & $P V_{1}$ & $\mathrm{PV}_{2}$ & $\mathbf{P V}_{3}$ & $\mathbf{P V}_{4}$ & $\mathrm{PV}_{5}$ & $\mathrm{PV}_{6}$ & \multirow[b]{3}{*}{ Acc. } & \multirow[b]{3}{*}{ Cov. } & \multirow[b]{3}{*}{ Fit. } \\
\hline & D & $\mathbf{N}$ & $\mathbf{N}$ & D & $\mathbf{N}$ & $\mathbf{N}$ & $\mathbf{N}$ & & & \\
\hline & $\begin{array}{l}\{1- \\
15\}\end{array}$ & $\begin{array}{c}\{1- \\
4\}\end{array}$ & $\begin{array}{c}\{1- \\
6\}\end{array}$ & $\begin{array}{l}\{1- \\
20\}\end{array}$ & $\begin{array}{l}\{1- \\
3\}\end{array}$ & $\begin{array}{l}\{1- \\
6\}\end{array}$ & $\begin{array}{c}\{1- \\
9\}\end{array}$ & & & \\
\hline 1 & $\{5-8\}$ & $\{2,4\}$ & $\{1\}$ & $\{1-2\}$ & - & - & - & $100 \%$ & $17 \%$ & $\begin{array}{l}1.7 \mathrm{x} \\
10^{-29}\end{array}$ \\
\hline 2 & - & - & - & - & $\{2\}$ & $\{4\}$ & $\{9\}$ & $100 \%$ & $26 \%$ & $\begin{array}{c}2.6 \mathrm{x} \\
10^{-46}\end{array}$ \\
\hline
\end{tabular}

For synthetic dataset 2, we ran 25 repetitions of the evolutionary algorithm (Algorithm 1). The algorithm was stopped when both the known a priori multivariate interactions were discovered and the number of generations and fitness evaluations were recorded. Again, the archived conjunctive clauses from the first iteration of the evolutionary algorithm were run through the GA (Algorithm 4) to find the best DNF.

\subsubsection{Real-World Chagas Dataset}

From August to October 2011, teams comprised of personnel from the Escuela de Biología, La Universidad de San Carlos Guatemala and the Guatemalan Ministry of Health Office of Vector-Borne Diseases conducted entomological and socioeconomic surveys of 1,132 houses in five villages in Olopa, Chiquimula, Guatemala. Informed consent was obtained from all human adult participants and from parents or legal guardians of minors. This project received ethical clearance from the Ministry of Health in Guatemala, La Universidad de San Carlos bioethics committee, and the Panamerican Health Organization. This Chagas dataset is not publicly available since it is human subject research data that is not possible to anonymize. The surveys contain 61 risk factors that experts believe are associated with infestation of households with Triatoma dimidiata, a vector of Chagas disease. Thirteen of the risk factors are ordinal/continuous and the remaining 48 are nominal. The total number of houses that are infested is $314(28 \%)$. Sixteen percent of the data are not present and are considered missing (rather than imputed). For the Chagas infestation dataset, the evolutionary algorithm (Algorithm 1) was run once for 1,500 generations to seek associations with households that are infested. Further analysis of the archived significant conjunctions for this dataset is beyond the scope of this paper and remains a subject for future study.

\section{RESULTS}

\subsection{Simulated Data Results}

An exhaustive search of the [21] dataset (for up to 20-way interactions) found over $10^{9}$ possible feature combinations present in the observations. Of the $\sim 10^{9}$ feature combinations, only 1,270 are correlated with having the disease and have a fitness score (by Eq. 1) less than the user defined threshold of $10^{-9}$; three of these were of order nine. 
Algorithm 1 found over $90 \%$ of the 1,270 conjunctive clauses that have a strong correlation with each output category in under 1,000 generations, in all 50 repetitions (Table 5). All four of the "true" bivariate feature combinations designed to correlate with the disease (from Table 1) were identified and archived in under 143,000 fitness evaluations; note this is significantly less than an exhaustive search of all $10^{9}$ possible conjunctive clauses. There are over $1.4 \times 10^{8}$ possible 9-way interactions in the dataset; yet in each run, Algorithm 1 was able to find at least one of the 3 significant 9way interactions (fitness $<10^{-9}$ ) in less than $1.4 \times 10^{5}$ evaluations. Thus the algorithm can find high order signals quickly in large search spaces (Table 5).

Table 5. Summary statistics for 50 repetitions of Algorithm 1 on synthetic dataset 1 (from [21]) showing the mean, minimum, and maximum number of generations (fitness evaluations are in parenthesis).

\begin{tabular}{|l|c|c|c|}
\cline { 2 - 4 } \multicolumn{1}{c|}{} & Mean & Min & Max \\
\hline $\begin{array}{l}\mathbf{4} \text { "true" } \\
\text { Clauses } \\
\text { (Table 1) }\end{array}$ & $\begin{array}{c}138 \\
(43,169)\end{array}$ & $\begin{array}{c}36 \\
(6,799)\end{array}$ & $\begin{array}{c}391 \\
(142,229)\end{array}$ \\
\hline $\begin{array}{l}\mathbf{7 5} \% \text { of all } \\
\text { Clauses }<\boldsymbol{T}\end{array}$ & 342 & 297 & 428 \\
$(124,263)$ & $(105,629)$ & $(157,665)$ \\
\hline $\begin{array}{l}\text { 90 \% of all } \\
\text { Clauses }<\boldsymbol{T}\end{array}$ & 602 & 510 & 720 \\
$(228,900)$ & $(191,896)$ & $(277,990)$ \\
\hline
\end{tabular}

The GA (Algorithm 4) consistently found the optimal DNF, which is the disjunction of the four conjunctions shown in Table 1, in each of the 50 repetitions. The GA was able to find this DNF after an average of 2,796 generations $(\min =380, \max =13,906)$, which corresponds to an average of 135,905 fitness evaluations $(\mathrm{min}=$ $17,734, \max =692,745)$. This is quite efficient, considering that there are over $1 \times 10^{43}$ first to twenty order (i.e., 1 to $\operatorname{Max}_{c c}$ ) possible combinations of conjunctive clauses.

For our synthetic dataset 2, Algorithm 1 archived both "true" 3-way and 4-way conjunctive clauses (shown in Table 4) designed to correlate with the output class $=1$ (see results in Table 6) in all 25 repetitions. This was a more difficult task than analyzing synthetic dataset 1 , since synthetic dataset 2 has features with larger ranges, was missing $20 \%$ of its data, the "true" interactions were order 3 and 4 , and the feature ranges/sets needed to be co-evolved. There are over $4.7 \times 10^{9} 3$-way feature combinations in the dataset, yet in 25 repetitions the algorithm was able to consistently find the exact "true" 3-way conjunction (from Table 4) while evaluating less than $2 \times 10^{5}$ conjunctive clauses of varying orders (Table 6). Similarly, there are $\sim 2.9 \times 10^{12}$ possible 4-way interactions in the dataset; but over 25 repetitions the algorithm was able to find the more complex "true" 4-way interaction (from Table 4) in less than $10^{7}$ fitness evaluations (Table 6).

In synthetic dataset 2 , the most-fit evolved DNF has an $\overline{A c c}=$ $0.655, \overline{\operatorname{Cov}}=0.457$, NonRep $=0.907$, and TotCov $=0.829$ and a fitness score of 0.225 (by Eq. 3). The best evolved DNF is given below:

$$
P V_{3} \in\{1-3\} \vee P V_{5} \in\{4\}
$$

The best evolved DNF contains two of the "true" features present in Table 4. The disjunction of the two "true" clauses has an $\overline{A c c}=$ $1.00, \overline{\operatorname{Cov}}=0.217$, NonRep $=1.000$, and TotCov $=0.434$ and an inferior fitness score of only 0.094 (by Eq. 3). The disparity in fitness is a result of the lower coverage in the two "true" clauses because we introduced a large amount of missing data to the dataset. Without the missing data, the DNF of the two "true" clauses has a fitness of 0.500 . Also, we did not guarantee that lower-order interactions or main effects were not correlated to the target class, as was done in synthetic dataset 1 .

Table 6. Summary statistics for 25 repetitions of Algorithm 1 on synthetic dataset 2, showing the mean, minimum, and maximum number of generations (fitness evaluations are in parenthesis) for finding both the 3-way and 4-way interactions in Table 4.

\begin{tabular}{|l|c|c|c|}
\multicolumn{1}{c|}{} & Mean & Min & Max \\
\hline Rule 1 & $\begin{array}{c}2,390 \\
(1,189,541)\end{array}$ & $\begin{array}{c}170 \\
(69,440)\end{array}$ & $\begin{array}{c}12,910 \\
(6,498,017)\end{array}$ \\
\hline \multirow{2}{*}{ Rule 2 } & 102 & 15 & 354 \\
& $(38,347)$ & $(2,868)$ & $(159,482)$ \\
\hline
\end{tabular}

\subsection{Chagas Data Results}

The evolutionary algorithm archived 178,719 conjunctive clauses associated with households infested with the Chagas vector (up to 5 -way interactions are shown in Figure 2). Only 11 of the 61 features are present in archived univariate conjunctive clauses. However, 46, 55, 57, 60 and 61 features are present in archived 2-, 3-, 4-, 5-, and 6-way interactions, respectively. Not surprisingly, the lower-order interactions and main effects tend to have higher coverage and lower accuracy, while high-order interactions tend to have higher accuracy and lower coverage (Figure 2). Figure 2 shows that 2-way interactions have higher accuracy and coverage than main affects at every point in the range except at the very highest levels of coverage. Also 3-, 4-, and 5-way interactions have higher accuracy and coverage than main effects at every point below $90 \%$ coverage. The plurality of the conjunctive clauses have 8 -way interactions and the evolutionary algorithm was even able to find one 22 -way interaction with $67 \%$ accuracy and $12 \%$ coverage. Note that one cannot infer dominance of the conjunctive clauses shown in Figure 2, since different clauses cover different parts of the space.

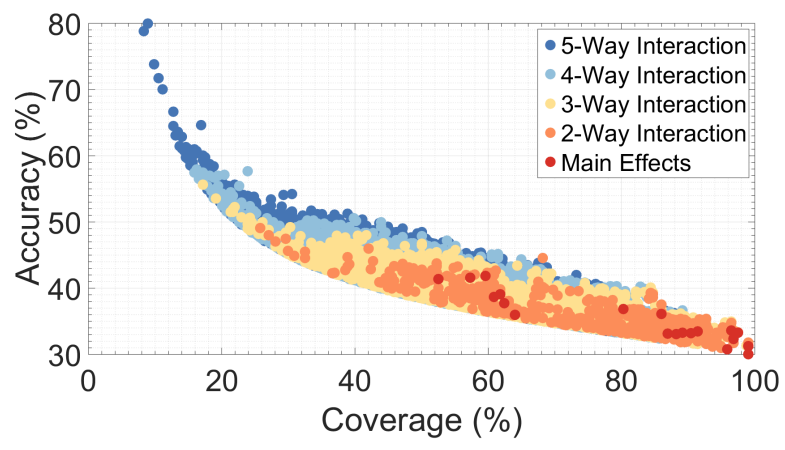

Figure 2. Coverage and accuracy of the 178,719 archived interactions associated with household infestation. Lower order conjunctive clauses are plotted on top of higher order.

\section{CONCLUSIONS}

We demonstrated a new methodology for efficiently searching for probabilistically significant multivariate interactions in complex heterogeneous "Big Datasets." The algorithm was validated on two synthetic datasets, one that contained purely epistatic bivariate interactions and another that contained a 3-way and a 4-way 
interaction, in addition to other complexities (nominal and discrete input features, 3- and 4-way interactions, missing data, heterogeneous associations with the same output class, and imbalanced class outcomes). Further experimentation has shown that lowering the probability threshold for the archive reduces the computational time for the evolutionary algorithm to find the true interactions in these synthetic datasets. However, in practice this comes with the risk of eliminating true signals for underrepresented segments of observations. We applied the method to a complex real-world Chagas infestation survey dataset and were able to archive many strong multivariate interactions. However, further analysis and interpretation of this set of archived conjunctions for the Chagas dataset is beyond the scope of this paper and remains a subject for future study.

\section{ACKNOWLEDGEMENTS}

We would like to thank researchers at La Universidad de San Carlos Guatemala, especially Carlota Monroy and Dulce Bustamante, for their help in collecting and managing the Chagas infestation dataset. We also thank the Guatemalan Ministry of Health for their help with collecting the data. This work was supported, in part, by Vermont EPSCoR with funds from the National Science Foundation Grant DBC-EID-1216193 (JPH) and EPS-1101317 (DMR).

\section{REFERENCES}

[1] Bustamante Zamora, D. M., Hernández, M. M., Torres, N., Zúniga, C., Sosa, W., de Abrego, V. \& Monroy Escobar, M. C. Information to Act: Household Characteristics are Predictors of Domestic Infestation with the Chagas Vector Triatoma dimidiata in Central America. American Journal of Tropical Medicine and Hygiene 93, 97-107 (2015).

[2] Butz, M. V. Rule-based evolutionary online learning systems a principled approach to LCS analysis and design. (Springer, 2006). at <http://site.ebrary.com/id/10143394>

[3] Control of Chagas disease second report of a WHO expert committee. (2002). at <http://site.ebrary.com/id/10040305>

[4] De Andrade, A. L., Zicker, F., De Oliveira, R.M., Da Silva,, I.G., Silva, S. A., De Andrade, S. S. \& Martelli, C. M. Evaluation of risk factors for house infestation by Triatoma infestans in Brazil. Am. J. Trop. Med. Hyg. 53, 443-447 (1995).

[5] De Jong, K. A. \& Spears, W. M. Learning concept classification rules using genetic algorithms. Proceedings of the Twelfth International Joint Conference on Artificial Intelligence, 651-656 (1991).

[6] De Jong, K. A., Spears, W. M. \& Gordon, D. F. Using genetic algorithms for concept learning. Machine Learning 13, 161-188 (1993).

[7] DeHaas, D., Craig, J., Rickert, C., Haake, P., Stor, K. \& Eppstein, M. J. Feature selection and classification in noisy epistatic problems using a hybrid evolutionary approach. In Proceedings of the 9th annual conference on Genetic and evolutionary computation, 1872-1872 (2007).

[8] Eiben, A. E. \& Smith, J. E. Introduction to evolutionary computing. (Springer, 2010).
[9] Enger, K. S., Ordoñez, R., Wilson, M. L. \& Ramsey, J. M. Evaluation of risk factors for rural infestation by Triatoma pallidipennis (Hemiptera: Triatominae), a Mexican vector of Chagas disease. J. Med. Entomol. 41, 760-767 (2004).

[10] Estimación cuantitativa de la enfermedad de Chagas en las Américas. (2006). at $<$ http://www.bvsops.org.uy/pdf/chagas19.pdf>

[11] Hornby, G. S. ALPS: the age-layered population structure for reducing the problem of premature convergence. In Proceedings of the 8th annual conference on Genetic and evolutionary computation, 815-822 (2006).

[12] Kendall, M. G. The advanced theory of statistics. (Hafner Publishing Company, 1952).

[13] Lin, M., Lucas, H. C. \& Shmueli, G. Research Commentary - Too Big to Fail: Large Samples and the $p$-Value Problem. Information Systems Research 24, 906-917 (2013).

[14] Lopes, C., Pacheco, M., Vellasco, M. \& Passos, E. in New Directions in Rough Sets, Data Mining, and Granular-Soft Computing (eds. Zhong, N., Skowron, A. \& Ohsuga, S.) 1711, 458-462 (Springer Berlin Heidelberg, 1999).

[15] Lucero, D. E., Morrissey, L. A., Rizzo, D. M., Rodas, A., Garnica, R., Stevens, L., Bustamante, D. M. \& Monroy, M. C. Ecohealth Interventions Limit Triatomine Reinfestation following Insecticide Spraying in La Brea, Guatemala. American Journal of Tropical Medicine and Hygiene 88, 630-637 (2013).

[16] Schmidt, M. D. \& Lipson, H. Age-fitness pareto optimization. In Proceedings of the 12th annual conference on Genetic and evolutionary computation, 543-544 (ACM Press, 2010).

[17] Stockwell, D. R. B. \& Noble, I. R. Induction of sets of rules from animal distribution data: A robust and informative method of data analysis. Mathematics and Computers in Simulation 33, 385-390 (1992).

[18] Stockwell, D. \& Peters, David. The GARP modelling system: problems and solutions to automated spatial prediction. International Journal of Geographical Information Science 13, 143-158 (1999).

[19] Urbanowicz, R. J., Bertasius, G. \& Moore, J. H. An extended Michigan-style learning classifier system for flexible supervised learning, classification, and data mining. In Parallel Problem Solving from Nature - PPSN XIII (eds. Bartz-Beielstein, T., Branke, J., Filipič, B. \& Smith, J.) 8672, 211-221 (Springer International Publishing, 2014).

[20] Urbanowicz, R. J., Granizo-Mackenzie, A. \& Moore, J. H. An analysis pipeline with statistical and visualization-guided knowledge discovery for Michigan-style learning classifier systems. IEEE Computational Intelligence Magazine 7, 3545 (2012).

[21] Urbanowicz, R. J. \& Moore, J. H. The application of Michigan-style learning classifier systems to address genetic heterogeneity and epistasis in association studies. In Proceedings of the 12th annual conference on Genetic and evolutionary computation, 195-210 (ACM Press, 2010). 